\title{
Radiation Dose Reduction in Paranasal Sinus CT Using Model-Based Iterative Reconstruction
}

\author{
J.M. Hoxworth, D. Lal, G.P. Fletcher, A.C. Patel, M. He, R.G. Paden, and A.K. Hara
}

\begin{abstract}
BACKGROUND AND PURPOSE: CT performed with Veo model-based iterative reconstruction has shown the potential for radiationdose reduction. This study sought to determine whether Veo could reduce noise and improve the image quality of low-dose sinus CT.
\end{abstract}

MATERIALS AND METHODS: Twenty patients consented to participate and underwent low- and standard-dose sinus CT on the same day. Standard-dose CT was created with filtered back-projection (120 kV[peak], $210 \mathrm{~mA}, 0.4$-second rotation, and 0.531 pitch). For low-dose $\mathrm{CT}, \mathrm{mA}$ was decreased to 20 (the remaining parameters were unchanged), and images were generated with filtered back-projection and Veo. Standard- and low-dose datasets were reconstructed by using bone and soft-tissue algorithms, while the low-dose Veo reconstruction only had a standard kernel. Two blinded neuroradiologists independently evaluated the image quality of multiple osseous and soft-tissue craniofacial structures. Image noise was measured by using multiple regions of interest.

RESULTS: Eight women and 12 men (mean age, 63.3 years) participated. Volume CT dose indices were 2.9 mGy (low dose) and $31.6 \mathrm{mGy}$ (standard dose), and mean dose-length products were $37.4 \mathrm{mGy}-\mathrm{cm}$ (low dose) and $406.1 \mathrm{mGy}-\mathrm{cm}$ (standard dose). Of all the imaging series, low-dose Veo demonstrated the least noise $(P<.001)$. Compared with filtered back-projection low-dose $C T$ using soft-tissue and bone algorithms, Veo had the best soft-tissue image quality but the poorest bone image quality $(P<.001)$.

CONCLUSIONS: Veo significantly reduces noise in low-dose sinus CT. Although this reduction improves soft-tissue evaluation, thin bone becomes less distinct.

ABBREVIATIONS: BONE FBP LD = bone algorithm, filtered back-projection, low dose; BONE FBP SD = bone algorithm, filtered back-projection, standard dose; IRIS = iterative reconstruction in image space; SAFIRE = sinogram-affirmed iterative reconstruction; SOFT FBP LD = soft-tissue algorithm, filtered back-projection, low dose; SOFT FBP SD = soft-tissue algorithm, filtered back-projection, standard dose; VEO LD = Veo model-based iterative reconstruction, low dose

A number of radiation dose-reduction strategies have been successfully used for paranasal sinus CT. Because radiation is unavoidably transmitted to the lens of the eye, orbital bismuth shielding has been used to reduce lens radiation exposure with minimal impact on image quality. ${ }^{1,2}$ Greater attention has been directed toward adjusting CT parameters, most commonly through the reduction of milliampere-second ( $\mathrm{mAs}$ ) (tube current time product), to allow reduced radiation exposure while maintaining acceptable image quality. ${ }^{3-13}$ Recently, high-pitch dual source multidetector CT systems have shown promise of even greater dose reduction. ${ }^{14,15}$ Nevertheless, because of pro-

Received July 9, 2013; accepted after revision August 11.

From the Departments of Radiology (J.M.H., G.P.F., A.C.P., R.G.P, A.K.H.) and Otolaryngology (D.L.), Mayo Clinic, Phoenix, Arizona; and School of Computing, Informatics, and Decision Systems Engineering, (M.H.), Arizona State University, Tempe, Arizona.

Please address correspondence to Joseph M. Hoxworth, MD, Department of Radiology, Mayo Clinic, 5777 E Mayo Blvd, Phoenix, AZ 85054; e-mail:

hoxworth.joseph@mayo.edu

http://dx.doi.org/10.3174/ajnr.A3749 gressively decreasing signal-to-noise, a threshold for tube output is invariably reached, below which imaging becomes unacceptable.

With the large computational capacities now available on normal workstations, iterative reconstruction techniques have emerged in CT as a viable alternative to the standard algorithm of filtered back-projection. Through increased complexity with more precise modeling of the acquisition process and the incorporation of various physical models, image quality can be maintained even at progressively lower radiation doses. ${ }^{16}$ Indeed, one such technique known as iterative reconstruction in image space (IRIS; Siemens, Erlangen, Germany) allows significant radiationdose reduction in sinus CT without compromising the image quality. ${ }^{17}$ More recently, sinogram-affirmed iterative reconstruction (SAFIRE; Siemens) demonstrated effective noise reduction in sinus CT at the expense of some image quality degradation. ${ }^{18}$ Unfortunately, because of the proprietary and vendor-specific nature of the iterative reconstruction techniques, results cannot be directly extrapolated across different CT platforms. As a result, 
Table 1: Image-noise measurement ${ }^{a}$

\begin{tabular}{lccccc}
\hline & $\begin{array}{c}\text { Pons } \\
(\boldsymbol{n}=\mathbf{2 0})\end{array}$ & $\begin{array}{c}\text { Globe } \\
(\boldsymbol{n}=\mathbf{2 0})\end{array}$ & $\begin{array}{c}\text { Masseter } \\
(\boldsymbol{n}=\mathbf{2 0})\end{array}$ & $\begin{array}{c}\text { Maxillary Sinus } \\
(\boldsymbol{n}=\mathbf{2 0})\end{array}$ & $\begin{array}{c}\text { Total } \\
(\boldsymbol{n}=\mathbf{8 0})\end{array}$ \\
\hline SOFT FBP LD & $74.9(10.60)^{\mathrm{b}}$ & $55.6(8.79)^{\mathrm{b}}$ & $52.6(8.79)^{\mathrm{b}}$ & $45.3(13.16)^{\mathrm{b}}$ & $57.1(15.06)^{\mathrm{b}}$ \\
BONE FBP LD & $278.8(36.73)^{\mathrm{b}}$ & $173.0(19.66)^{\mathrm{b}}$ & $175.3(27.03)^{\mathrm{b}}$ & $105.5(15.64)^{\mathrm{b}}$ & $183.1(67.38)^{\mathrm{b}}$ \\
VEO LD & $18.9(2.98)$ & $12.4(1.37)$ & $14.3(2.16)$ & $12.3(2.11)$ & $14.5(3.49)$ \\
SOFT FBP SD & $24.7(5.14)^{\mathrm{b}}$ & $19.5(3.06)^{\mathrm{b}}$ & $19.1(3.35)^{\mathrm{b}}$ & $17.5(2.48)^{\mathrm{b}}$ & $20.2(4.48)^{\mathrm{b}}$ \\
BONE FBP SD & $97.4(14.47)^{\mathrm{b}}$ & $66.9(7.78)^{\mathrm{b}}$ & $66.3(8.96)^{\mathrm{b}}$ & $50.1(6.42)^{\mathrm{b}}$ & $70.2(19.73)^{\mathrm{b}}$ \\
\hline
\end{tabular}

${ }^{a}$ Values are expressed as mean (SD) of image noise measured in Hounsfield units.

b Statistically significant difference $(P<.001)$ in paired comparison with VEO LD.

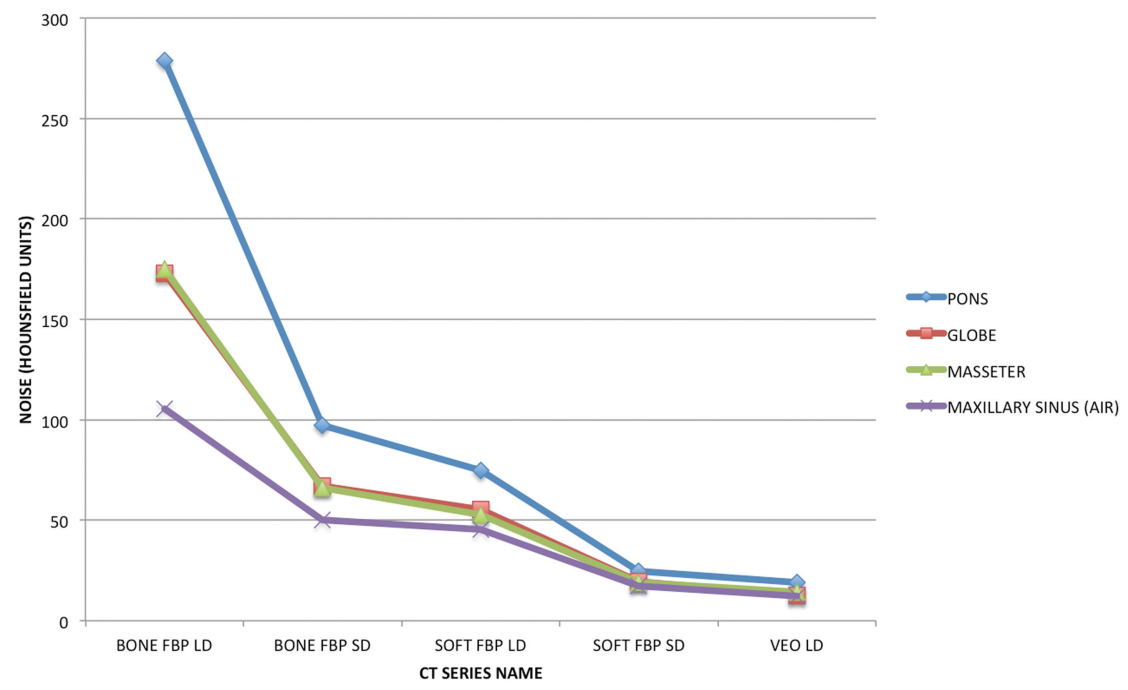

FIG 1. Graphic representation of mean image noise for the pons, globe, masseter, and air within the maxillary sinus for each of the tested CT series: soft-tissue algorithm, filtered back-projection, low dose; bone algorithm, filtered back-projection, low dose; Veo model-based iterative reconstruction, low dose; soft-tissue algorithm, filtered back-projection, standard dose; and bone algorithm, filtered back-projection, standard dose.

the objective of this study was to compare low-radiation-dose sinus CT processed with Veo model-based iterative reconstruction (GE Healthcare, Milwaukee, Wisconsin) with standard and low-dose sinus CT generated with filtered back-projection through the evaluation of image noise and diagnostic image quality in 20 patients.

\section{MATERIALS AND METHODS}

This Health Insurance Portability and Accountability Actcompliant study was approved by the Mayo Clinic Institutional Review board, and all patients provided written informed consent. Twenty patients 18 years of age or older who were previously scheduled for a clinically indicated sinus CT were invited to participate. Individuals with a history of previous sinonasal surgery or a known maxillofacial neoplasm were specifically excluded.

Noncontrast paranasal sinus CT was performed on all study participants by using a 64-detector row multidetector CT scanner (Discovery CT750 HD, GE Healthcare) with the patient in the supine position, extending from the hard palate superiorly to include the frontal sinuses. Standard-dose sinus CT was performed by using $120 \mathrm{kV}$ (peak), $210 \mathrm{~mA}, 0.4$-second rotation time, 0.531 pitch, and $0.625-\mathrm{mm}$ section collimation; images were generated by using filtered back-projection. Next, a low-dose sinus CT was acquired by using the same parameters except that the $\mathrm{mA}$ were reduced to 20 . Low-dose images were generated by using both filtered back-projection and Veo model-based iterative reconstruction. Low- and standard-dose datasets from filtered back-projection were reconstructed by using bone (GE kernel, bone) and soft-tissue (GE kernel, standard) algorithms, while low-dose CT was also reconstructed with Veo (note that Veo only offered a single standard kernel). As a result, 5 imaging series were created for each patient: 1) soft-tissue algorithm, filtered back-projection, low dose [SOFT FBP LD], 2) bone algorithm, filtered back-projection, low dose [BONE FBP LD], 3) Veo modelbased iterative reconstruction, low dose [VEO LD], 4) soft-tissue algorithm, filtered back-projection, standard dose [SOFT FBP SD], and 5) bone algorithm, filtered back-projection, standard dose [BONE FBP SD].

Each image set was transferred to an AW Workstation (Version 4.2; GE Healthcare) in an anonymized fashion, where it was reformatted at $1-\mathrm{mm}$ increments in the axial and coronal planes. Using a round region of interest with a diameter of $1 \mathrm{~cm}$, a single blinded observer measured the mean attenuation and SD in Hounsfield units of the central pons, right globe (vitreous), right masseter muscle, and air within the center of the right maxillary sinus in the axial plane. The SD was used to represent image noise. For the evaluation of diagnostic image quality, 2 board-certified neuroradiologists independently reviewed the imaging series blinded to all patient identifiers and CT parameters. Specifically, they graded image quality for the nasal septum, middle turbinate, lamina papyracea, cribriform plate, optic nerve, inferior rectus muscle, globe (eye), and brain on a scale from 1 (unacceptable noise, nondiagnostic) to 5 (excellent image quality, best diagnostic value). For each of the 5 series, the reviewers were limited to 2 preset window level and width settings for emphasis on bone (window level, $800 \mathrm{HU}$; window width, $3500 \mathrm{HU}$ ) and soft tissue (window level, $40 \mathrm{HU}$; window width, $400 \mathrm{HU}$ ). Last, an experienced endoscopic sinus surgeon was asked to qualitatively assess the low-dose sinus CT scans without and with VEO LD to determine whether there was sufficient anatomic detail for preoperative planning, and this assessment was recorded in a binary fashion as yes or no.

Statistical analyses were performed by using SAS, Version 9.2 (SAS Institute, Cary, North Carolina). For descriptive analysis, the image-quality score and noise level of different imaging series by structure were presented as mean and SD; the differences among structures were assessed by the Kruskal-Wallis test. Separate mixed models were developed for image quality and image noise. For the image noise mixed model, 5 imaging series and 4 structures were included as fixed effects, and the interaction term 
Table 2: Image-quality assessment ${ }^{\mathrm{a}}$

\begin{tabular}{|c|c|c|c|c|c|c|c|c|c|}
\hline & $\begin{array}{l}\text { Brain } \\
(n=40)\end{array}$ & $\begin{array}{l}\text { Globe } \\
(n=40)\end{array}$ & $\begin{array}{c}\text { Inferior Rectus } \\
\text { Muscle } \\
(n=40)\end{array}$ & $\begin{array}{l}\text { Optic } \\
\text { Nerve } \\
(n=40)\end{array}$ & $\begin{array}{l}\text { Cribriform } \\
\text { Plate } \\
(n=40)\end{array}$ & $\begin{array}{l}\text { Lamina } \\
\text { Papyracea } \\
(n=40)\end{array}$ & $\begin{array}{l}\text { Middle } \\
\text { Turbinate } \\
(n=40)\end{array}$ & $\begin{array}{l}\text { Nasal } \\
\text { Septum } \\
(n=40)\end{array}$ & $\begin{array}{c}\text { Total } \\
(n=320)\end{array}$ \\
\hline SOFT FBP LD & $1.5(0.51)^{b}$ & $1.6(0.55)^{b}$ & $1.9(0.43)^{b}$ & $1.8(0.52)^{b}$ & $3.3(0.47)$ & $3.1(0.38)^{b}$ & $3.3(0.44)$ & $3.1(0.38)$ & $2.4(0.90)^{b}$ \\
\hline BONE FBP LD & $1.0(0.00)^{b}$ & $1.0(0.16)^{b}$ & $1.0(0.16)^{b}$ & $1.1(0.27)^{\mathrm{b}}$ & $3.7(0.62)^{\mathrm{b}}$ & $3.7(0.56)^{b}$ & $3.4(0.58)$ & $3.6(0.59)^{b}$ & $2.3(1.35)^{\mathrm{b}}$ \\
\hline VEO LD & $2.8(0.41)$ & $2.9(0.35)$ & $3.1(0.32)$ & $3.1(0.32)$ & $3.2(0.48)$ & $2.7(0.53)$ & $3.2(0.53)$ & $3.0(0.45)$ & $3.0(0.46)$ \\
\hline SOFT FBP SD & $3.4(0.55)^{\mathrm{b}}$ & $4.0(0.42)^{b}$ & $4.0(0.58)^{\mathrm{b}}$ & $4.1(0.40)^{\mathrm{b}}$ & $4.0(0.51)^{b}$ & $3.9(0.47)^{b}$ & $4.0(0.55)^{\mathrm{b}}$ & $3.9(0.56)^{b}$ & $3.9(0.54)^{b}$ \\
\hline BONE FBP SD & $2.0(0.28)^{b}$ & $2.5(0.51)^{b}$ & $3.0(0.53)$ & $3.1(0.45)$ & $4.7(0.47)^{\mathrm{b}}$ & $4.9(0.33)^{\mathrm{b}}$ & $4.7(0.46)^{\mathrm{b}}$ & $4.9(0.36)^{b}$ & $3.7(1.19)^{\mathrm{b}}$ \\
\hline
\end{tabular}

${ }^{a}$ Values are expressed as mean (SD). Image-quality scale ranges from 1 (unacceptable noise, nondiagnostic) to 5 (excellent image quality, best diagnostic value)

${ }^{b}$ Statistically significant difference $(P<.001)$ in paired comparison with VEO LD.

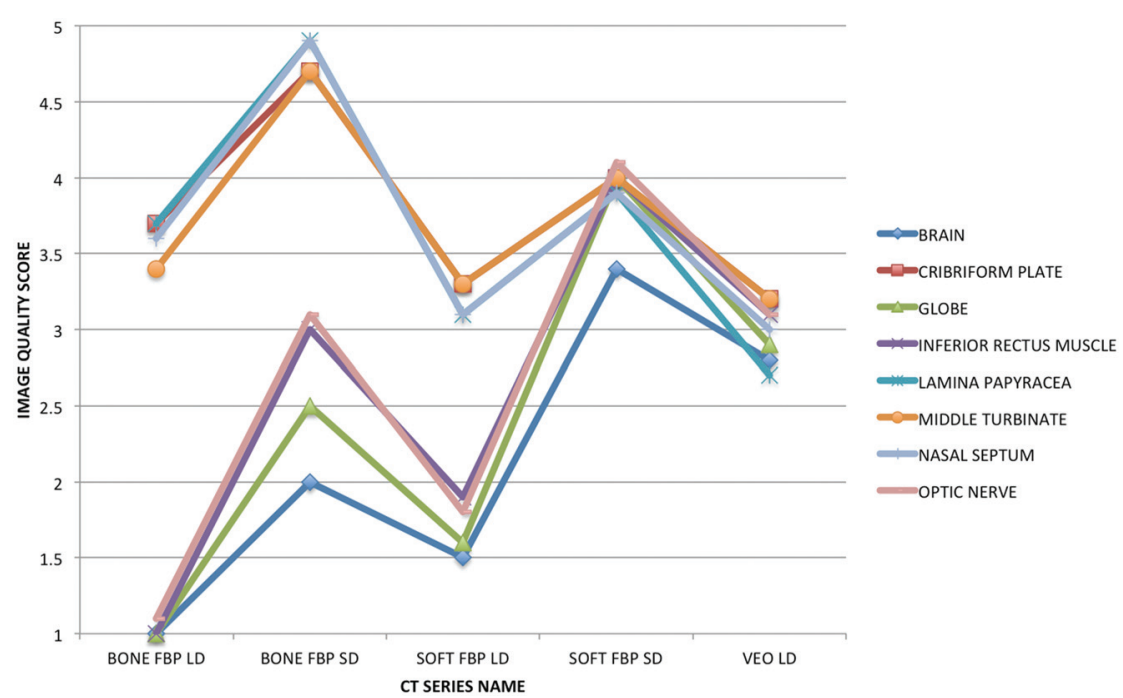

FIG 2. Graphic representation of mean image quality for the brain, cribriform plate, globe, inferior rectus muscle, lamina papyracea, middle turbinate, nasal septum, and optic nerve for each of the tested CT series: soft-tissue algorithm, filtered back-projection, low dose; bone algorithm, filtered back-projection, low dose; Veo model-based iterative reconstruction, low dose; soft-tissue algorithm, filtered back-projection, standard dose; and bone algorithm, filtered back-projection, standard dose.

for the "structure*imaging series" was significant and was included in the model. For the image quality mixed model, 5 imaging series, 2 readers, and 8 structures were included as fixed effects. The interaction term for the "structure*image" series was significant and was included in the model, while nonsignificant interaction terms such as "reader*structure" and "reader*image series" were removed from the final model. Random effects allowed covariance to vary due to subject. Post hoc paired $t$ tests were conducted to compare image quality scores and noise levels between each possible imaging series pair overall and for each subgroup of structures. $P$ values $<.05$ were considered statistically significant.

\section{RESULTS}

Twenty patients were recruited, and the study cohort consisted of 8 women and 12 men with an average age of $63.3 \pm 14.9$ years. Volume CT dose index means for LD and SD were $2.9 \pm 0 \mathrm{mGy}$ and $31.6 \pm 0 \mathrm{mGy}$, respectively, while the corresponding mean dose-length products were $37.4 \pm 2.5 \mathrm{mGy}-\mathrm{cm}$ and $406.1 \pm 25.9$ mGy-cm.

The results of noise quantification are summarized in Table 1 and graphically represented in Fig 1 . As expected, the use of a lower radiation dose and application of a bone algorithm resulted in an increase in image noise in instances in which images were generated by using filtered backprojection. However, despite a markedly reduced radiation dose, VEO LD demonstrated significantly less image noise compared with all other imaging series $(P<$ .001).

Image quality assessment is reported in Table 2 and illustrated in Fig 2. Compared with bone-containing structures (cribriform plate, lamina papyracea, middle turbinate, nasal septum), the soft-tissue structures (brain, optic nerve, globe, inferior rectus muscle) were suboptimally evaluated secondary to increased noise in the filtered back-projection series reconstructed with a bone algorithm and/or obtained with a low radiation dose (ie, BONE FBP SD, BONE FBP LD, SOFT FBP LD). The image quality for the softtissue and bone-containing structures converged in the SOFT FBP SD series with improved soft-tissue detail at the expense of mildly reduced image quality in the evaluation of bone-containing structures. A similar pattern of image quality convergence for bone and soft-tissue structures was observed in the VEO LD group, albeit at a significantly lower image quality compared with SOFT FBP SD, despite lower image noise. Representative examples from the 5 imaging series are presented in soft-tissue (Fig 3) and bone algorithms (Fig 4).

If patients were exclusively imaged by using the low-radiationdose technique for sinus $\mathrm{CT}$, the most relevant question is whether the noise reduction of VEO LD improves image quality. In an aggregate analysis of all structures, image quality was significantly improved in VEO LD compared with BONE FBP LD and SOFT FBP LD. However, in analyzing the results by structure, this benefit was derived from VEO LD outperforming BONE FBP LD and SOFT FBP LD in the evaluation of soft-tissue structures. In contrast, VEO LD generally had poorer image quality for the bone-containing structures (Table 2).

Irrespective of the inclusion of VEO LD, none of the lowdose sinus CT scans were graded as adequate for preoperative planning.

\section{DISCUSSION}

In contrast to reducing radiation exposure by modifying doserelated technical parameters within the confines of a filtered backprojection algorithm, new CT systems offer the potential for fur- 

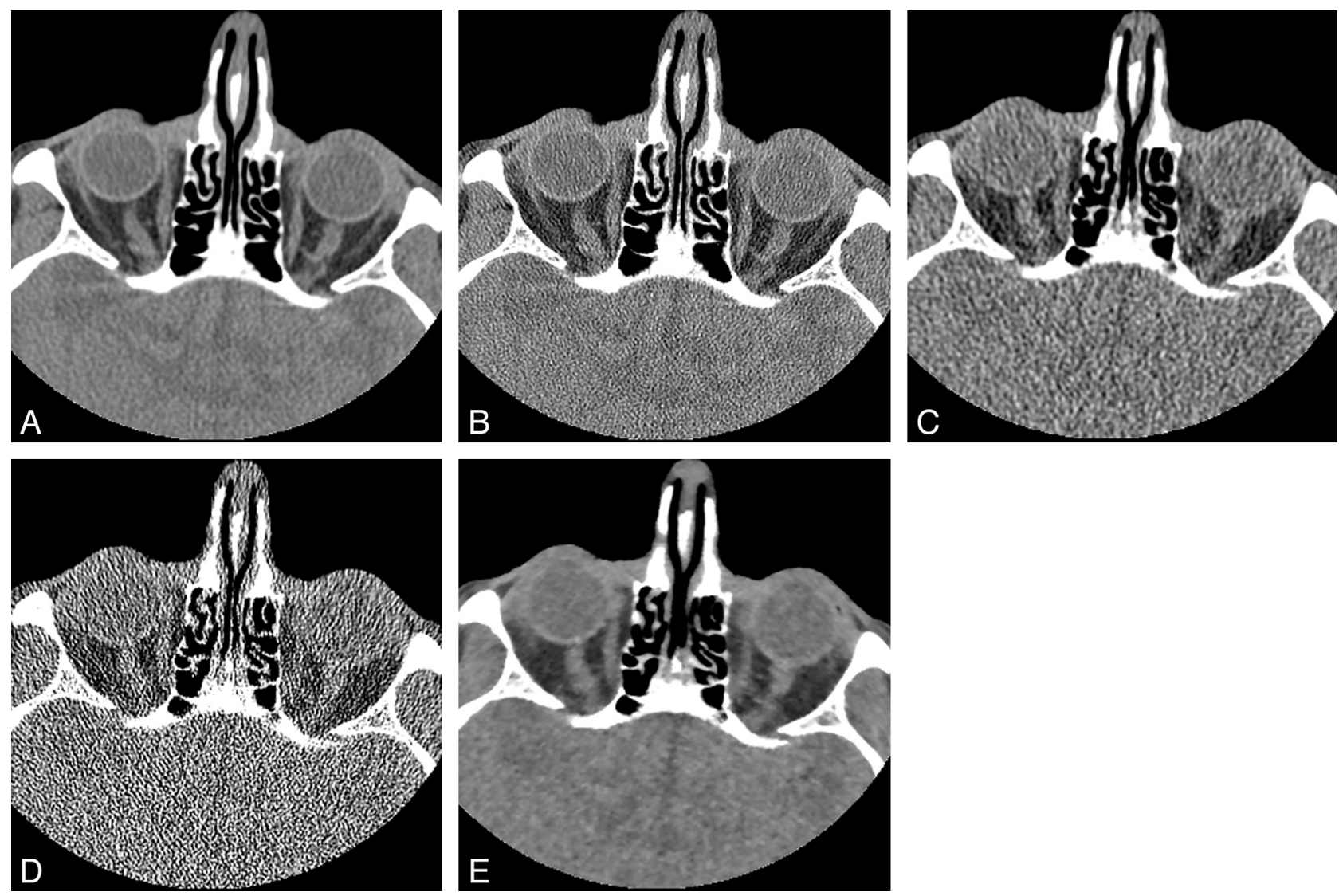

FIG 3. Axial noncontrast sinus $\mathrm{CT}$ (window level, $40 \mathrm{HU}$; window width, $400 \mathrm{HU}$ ) through the level of the orbits for the following imaging series: soft-tissue algorithm, filtered back-projection, standard dose $(A)$; bone algorithm, filtered back-projection, standard dose $(B)$; soft-tissue algorithm, filtered back-projection, low dose (C); bone algorithm, filtered back-projection, low dose (D); and Veo model-based iterative reconstruction, low dose $(E)$. The decreased image noise secondary to Veo allows improved visualization of orbital and intracranial soft-tissue structures relative to the other low-dose protocols but not to the level of the standard-dose sinus CT.

ther dose reduction while preserving image quality through the use of iterative reconstruction. ${ }^{16}$ Whether using statistical or model-based methods, these techniques proceed through an iterative loop in which a forward-projected image is compared with measured raw data and a correction factor is applied to the volumetric estimate, which is then back-projected. This iteration continues to converge toward a better solution until a predefined criterion for stopping is met. Unfortunately, the commercially available iterative reconstruction techniques from the major CT vendors must largely be viewed as a "black box," because radiation dose-reduction strategies allow a competitive advantage in the marketplace. Consequently, the performance of iterative reconstruction algorithms must be independently tested and validated in different body regions.

The use of Veo in low-dose sinus CT performed with a mean dose-length product of $37.4 \mathrm{mGy}$-cm significantly reduced image noise in comparison with filtered back-projection, and this reduction translated into improved image quality in the evaluation of craniofacial soft-tissue structures. Unfortunately, the smoothing effect of the Veo model-based iterative reconstruction impaired evaluation of structures containing thin bone at a low dose. Despite suboptimal performance for bone resolution, Veo may still prove to be a useful adjunct reconstruction technique for lowdose sinus CT. In evaluating the impact of radiation-dose reduction on multidetector sinus CT image quality, Brem et $\mathrm{al}^{4}$ found that soft tissues tolerate much less dose reduction compared with bone. Effective mAs could only be decreased to 134 to maintain diagnostic image quality for soft-tissue components, but diagnostic quality was maintained to an effective $\mathrm{mAs}$ of 67 if only osseous structures were considered. As a result, Veo may allow greater dose reduction for sinus CT by helping to salvage soft-tissue image quality.

To date, only 2 other studies have evaluated iterative reconstruction for potential radiation-dose reduction in the performance of sinus CT, both of which were developed by Siemens. Bulla et $\mathrm{al}^{17}$ incorporated IRIS into a low-dose sinus CT protocol that compared their standard-dose CT $(60 \mathrm{mAs}, 120 \mathrm{kV})$ with image datasets acquired at $48 \mathrm{mAs}, 36 \mathrm{mAs}$, and $24 \mathrm{mAs}$, through the evaluation of subjective image quality. Compared with filtered back-projection at $60 \mathrm{mAs}$, IRIS was found to improve image quality at $48 \mathrm{mAs}$, with no statistically significant decrement in image quality at the lower doses of 36 and $24 \mathrm{mAs}$. Notably, the authors still could reconstruct images in a bone kernel with IRIS, which is not an option with Veo tested in the current study. Because quantitative noise measurements were not made in the study of Bulla et al, it is not possible to draw any comparison of image quality and the degree of noise reduction. Schulz et al ${ }^{18}$ tested sinogram-affirmed iterative reconstruction by using a phantom head model at different radiation doses $(100-120 \mathrm{kV}$, 25-100 mAs). They evaluated image quality and measured noise, 

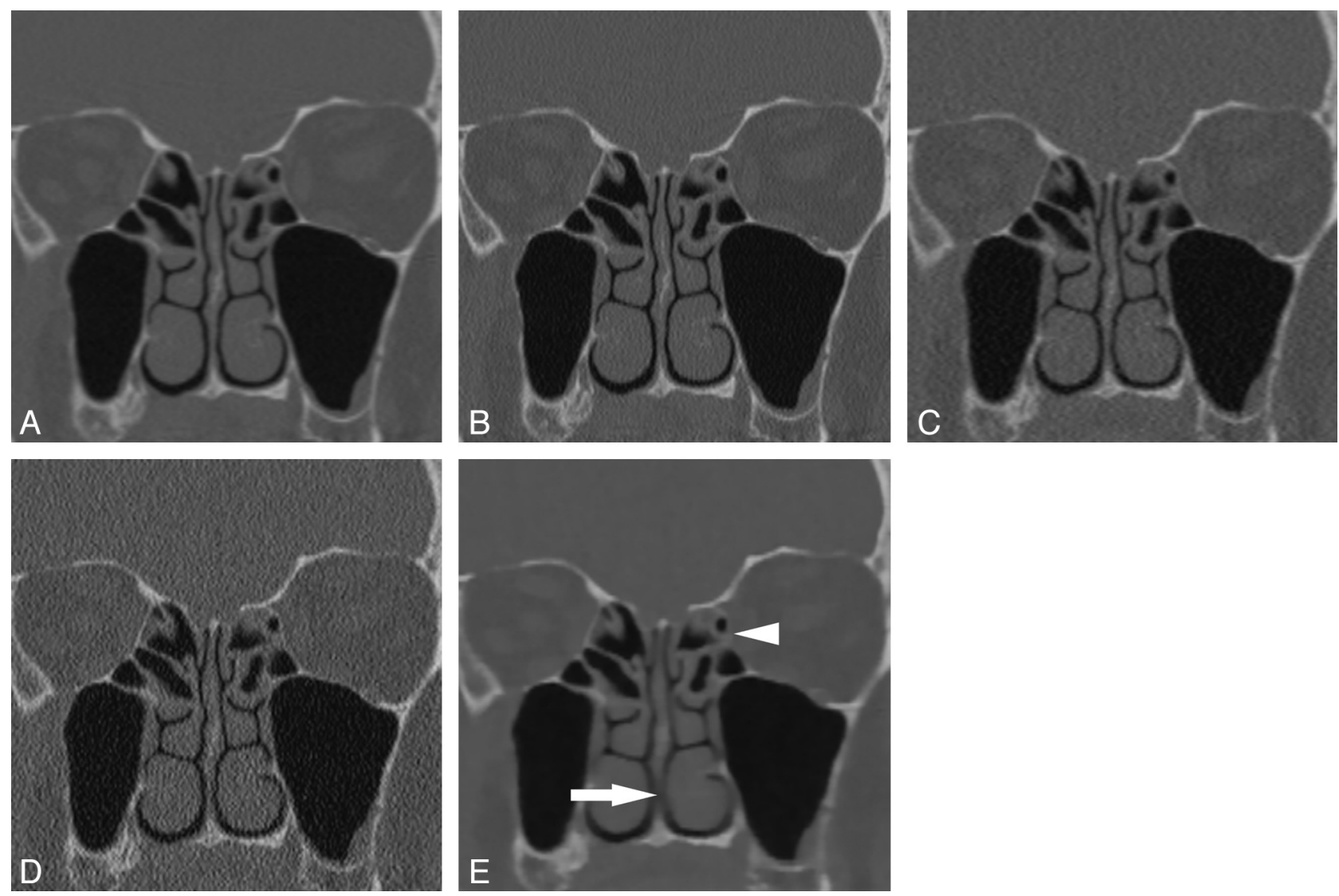

FIG 4. Coronal noncontrast sinus $\mathrm{CT}$ (window level, $800 \mathrm{HU}$; window width, $3500 \mathrm{HU}$ ) through the level of the maxillary sinuses for the following imaging series: soft-tissue algorithm, filtered back-projection, standard dose (A); bone algorithm, filtered back-projection, standard dose (B); soft-tissue algorithm, filtered back-projection, low dose (C); bone algorithm, filtered back-projection, low dose (D); and Veo model-based iterative reconstruction, low dose $(E)$. The noise reduction achieved with Veo results in loss of edge enhancement, making thin bones such as the nasal septum (white arrow) and left lamina papyracea (white arrowhead) difficult to see.

and comparison was made with both filtered back-projection and IRIS. The SAFIRE software permits 5 different levels of noise suppression (I-V), and these were tested by using both hard and soft kernels. SAFIRE V behaved closest to Veo in that it achieved the greatest degree of noise reduction (up to $85 \%$ ) but also received the lowest scores for image quality. The readers in that study preferred the highest tube current images rendered with filtered back-projection, even though the lowest setting of SAFIRE I also had less noise. The authors concluded that it may not be possible to compensate for insufficient photons through greater levels of iterative reconstruction because the latter may soften bone edges and cortical structures to an unacceptable level.

Wide variation exists in the radiation dose of sinus CT protocols at different institutions, largely because of the individual preferences of both radiologists and surgeons. ${ }^{19-21}$ Because no universally accepted standard exists for image quality, Hojreh et $\mathrm{al}^{6}$ proposed using image noise measured in a phantom as an indicator of appropriate sinus CT radiation dose. It was suggested that low-dose sinus CT be characterized by a pixel noise of 70-90 $\mathrm{HU}$, normal-dose sinus CT have a pixel noise of 50-70 HU, and high-dose sinus CT produce a pixel noise of $<50 \mathrm{HU}$. Although the objective and reproducible nature of this technique is appealing, the noise measurements must be referenced to an edge-enhancing reconstruction algorithm with filtered back-projection because the current study of Veo and previous work with SAFIRE reveal that lower noise cannot be equated to improved image quality for sinus CT by using iterative reconstruction. ${ }^{18}$

Previous studies have tested the impact of reducing the radiation dose to as low as a volume CT dose index of $1.1 \mathrm{mGy}$ on computer-assisted surgical navigation and have found no technical limitation on the surface registration algorithm or navigation accuracy. ${ }^{20,22}$ Instead, the level of dose reduction was dictated by the different needs for image quality of the individual surgeon, which are heavily driven by bone anatomic detail. In the current study, the inclusion of Veo did not convert any of the low-dose sinus CT scans from unacceptable to acceptable for preoperative planning. Compared with the bone algorithm reconstruction of a filtered back-projection low-dose CT, an iterative reconstruction technique would need to improve the bone detail of sinonasal landmarks to accomplish this conversion.

Limitations of this study include the small sample size, though the statistical power was sufficient to demonstrate key differences between Veo and filtered back-projection. Only a single low-dose sinus CT was performed in each patient, without the evaluation of additional tube current settings. The very low mAs used in this study was selected in an attempt to better elicit potential differences in image quality and noise between filtered back-projection and Veo. Although Veo improved the image quality of soft-tissue structures, the image quality was still suboptimal at this very low dose. A higher radiation dose, perhaps at $50 \%$ of the standard 
dose, would provide better image quality but would still significantly reduce the dose. However, it is likely that Veo would perform similarly at an intermediate radiation dose by reducing image noise without improving bone detail. Ultimately, radiation dose reduction in sinus CT must balance noise reduction and bone resolution. Future refinement of Veo for use in low-dose sinus CT should include the option of a high-resolution bone algorithm. This is currently not available with Veo but is included with some other commercially available adaptive statistical and model-based iterative reconstruction packages. Given that the VEO LD series had significantly lower image noise than all filtered back-projection imaging series regardless of radiation dose, it may be possible to sacrifice some noise reduction at the expense of improved edge enhancement.

\section{CONCLUSIONS}

Veo model-based iterative reconstruction significantly reduces image noise in low-dose sinus CT. As tested, this reduction caused some obscuration of fine detail of thin bone, and future versions of Veo should ideally include the option of a high-resolution bone algorithm. Veo improved visualization of soft-tissue structures, though not to a diagnostic level because of the very low radiation dose used in this study. Given that low-dose sinus CT performed with standard filtered back-projection still permits visualization of high contrast bone at the expense of soft-tissue image quality, the incorporation of Veo into a low-dose sinus imaging protocol may be useful for improving soft-tissue evaluation.

Disclosures: Joseph M. Hoxworth, Devyani Lal, Geoffrey P. Fletcher, Ameet C. Patel, Amy K. Hara, and Robert G. Paden-RELATED: Other: GE Healthcare, ${ }^{*}$ Comments: Through a research agreement with GE Healthcare, the Veo model-based iterative reconstruction system was installed on a single Discovery CT750 HD scanner and the cost was offset to $\$ 0$ through the performance of multiple research studies to assist with product development. GE Healthcare did not participate in any aspect of subject recruitment, data collection, data analysis, or manuscript preparation. Amy K. Hara-UNRELATED: Royalties: GE Healthcare, ${ }^{*}$ Comments: for CT colonography software. *Money paid to the institution.

\section{REFERENCES}

1. Perisinakis K, Raissaki M, Theocharopoulos N, et al. Reduction of eye lens radiation dose by orbital bismuth shielding in pediatric patients undergoing CT of the head: a Monte Carlo study. Med Phys 2005;32:1024-30

2. Hein E, Rogalla P, Klingebiel R, et al. Low-dose CT of the paranasal sinuses with eye lens protection: effect on image quality and radiation dose. Eur Radiol 2002;12:1693-96

3. Abul-Kasim K, Strombeck A, Sahlstrand-Johnson P. Low-dose computed tomography of the paranasal sinuses: radiation doses and reliability analysis. Am J Otolaryngol 2011;32:47-51

4. Brem MH, Zamani AA, Riva R, et al. Multidetector CT of the para- nasal sinus: potential for radiation dose reduction. Radiology 2007;243:847-52

5. Hagtvedt T, Aalokken TM, Notthellen J, et al. A new low-dose CT examination compared with standard-dose CT in the diagnosis of acute sinusitis. Eur Radiol 2003;13:976-80

6. Hojreh A, Czerny C, Kainberger F. Dose classification scheme for computed tomography of the paranasal sinuses. Eur J Radiol 2005;56:31-37

7. Kearney SE, Jones $\mathrm{P}$, Meakin K, et al. CT scanning of the paranasal sinuses-the effect of reducing mAs. Br J Radiol 1997;70:1071-74

8. Lam S, Bux S, Kumar G, et al. A comparison between low-dose and standard-dose non-contrasted multidetector CT scanning of the paranasal sinuses. Biomed Imaging Interv J 2009;5:e13

9. Mulkens TH, Broers C, Fieuws S, et al. Comparison of effective doses for low-dose MDCT and radiographic examination of sinuses in children. AJR Am J Roentgenol 2005;184:1611-18

10. Sohaib SA, Peppercorn PD, Horrocks JA, et al. The effect of decreasing $\mathrm{mAs}$ on image quality and patient dose in sinus CT. Br J Radiol 2001;74:157-61

11. Tack D, Widelec J, De Maertelaer V, et al. Comparison between lowdose and standard-dose multidetector CT in patients with suspected chronic sinusitis. AJR Am J Roentgenol 2003;181:939-44

12. Duvoisin B, Landry M, Chapuis L, et al. Low-dose CT and inflammatory disease of the paranasal sinuses. Neuroradiology 1991;33: 403-06

13. Marmolya G, Wiesen EJ, Yagan R, et al. Paranasal sinuses: low-dose CT. Radiology 1991;181:689-91

14. Schulz B, Potente S, Zangos S, et al. Ultra-low dose dual-source highpitch computed tomography of the paranasal sinus: diagnostic sensitivity and radiation dose. Acta Radiol 2012;53:435-40

15. Schell B, Bauer RW, Lehnert T, et al. Low-dose computed tomography of the paranasal sinus and facial skull using a high-pitch dualsource system-first clinical results. Eur Radiol 2011;21:107-12

16. Beister M, Kolditz D, Kalender WA. Iterative reconstruction methods in X-ray CT. Phys Med 2012;28:94-108

17. Bulla S, Blanke $\mathrm{P}$, Hassepass $\mathrm{F}$, et al. Reducing the radiation dose for low-dose CT of the paranasal sinuses using iterative reconstruction: feasibility and image quality. Eur J Radiol 2012;81:2246-50

18. Schulz B, Beeres M, Bodelle B, et al. Performance of iterative image reconstruction in $\mathrm{CT}$ of the paranasal sinuses: a phantom study. AJNR Am J Neuroradiol 2013;34:1072-76

19. Koller CJ, Eatough JP, Bettridge A. Variations in radiation dose between the same model of multislice CT scanner at different hospitals. Br J Radiol 2003;76:798-802

20. Nauer CB, Eichenberger A, Dubach P, et al. CT radiation dose for computer-assisted endoscopic sinus surgery: dose survey and determination of dose-reduction limits. AJNR Am J Neuroradiol 2009;30:617-22

21. Stewart AE, Stalp JT, Futerman C, et al. Variability of radiation exposure with in-office sinus computed tomography examinations. Int Forum Allergy Rhinol 2011;1:313-18

22. Dubach P, Eichenberger A, Caversaccio M. Radiation dose reduction in computer assisted navigation for functional endoscopic sinus surgery: cadaver head experiments and clinical implementation. Rhinology 2010;48:339-43 